\title{
Evaluation of patients with PFPS using a standardized Q angle measurement protocol
}

\author{
Adil Songur ${ }^{1} \oplus, *$, Ertuğrul Demirdel ${ }^{2} \oplus$ \\ ${ }^{1}$ Ankara Yıldırım Beyazıt University, Institute of Health Sciences. Turkey. ${ }^{2}$ Ankara Yıldırım Beyazıt University, Faculty of Health \\ Sciences, Turkey.
}

Abstract. An important concept in patellofemoral joint function is the quadriceps angle ( $Q$ angle), but there is a standardization problem with the measurement method. The aim of the study was to compare the change of the $\mathrm{Q}$ angle in relaxed or maximum voluntary contraction (MVC) of the quadriceps using a standardized measurement method in patients with patellofemoral pain syndrome (PFPS). This cross-sectional analytical study includes 34 sedentary female patients aged 18-50 years with a diagnosis of PFPS. Measurements were carried out with a manual extendable fixedarm goniometer. For the first measurement, the patient was placed in quadriceps relaxed in supine position. Lower extremity rotation was neutralized using the axial rotation neutralizing apparatus (ARNA). For the second measurement, while the quadriceps was maximally contracted in the same position the midpoint of the patella was re-marked and then the measurement was repeated. The $Q$ angle measurements taken in quadriceps relaxed and MVC were $18.44^{\circ} \pm 2.80^{\circ}$ and $16.41^{\circ} \pm 2.83^{\circ}$, respectively $(\mathrm{p}=.00)$. It was determined that there was no correlation between age, height, weight, body mass index (BMI), leg length and $\mathrm{Q}$ angle measurements of the participants $(p>.05)$. In conclusion, this measurement method offered measurement estimations that verified the effect of the quadriceps on the $Q$ angle in patients with PFPS. The prevention of axial rotation in the leg and the use of an extendable fixed-arm goniometer contribute to the standardization of $\mathrm{Q}$ angle measurement.

Keywords. ARNA, PFPS, Q angle, quadriceps.

\section{Introduction}

Patellofemoral pain syndrome (PFPS) is the most common musculoskeletal problem characterized by anterior knee pain in adults (Crossley et al., 2016). PFPS is 2.23 times higher in women compared to men (Boling et al., 2010). The clinical manifestation of PFPS is often associated with muscle weakness and altered lower extremity biomechanics (Santos et al., 2015).

The quadriceps angle ( $Q$ angle) is a parameter that gives important information about the patellofemoral joint alignment. The $\mathrm{Q}$ angle is a risk factor for patellofemoral pain, patellar subluxation, dislocation, and even anterior cruciate ligament injury. The $\mathrm{Q}$ angle is widely used to evaluate patients with knee problems especially cases with PFPS (Brechter \& Powers, 2002; Insall et al., 1976; Lee et al., 2003). The $Q$ angle measurement is traditionally performed by a goniometer in supine, knee extension, and quadriceps in a relaxed position (Insall et al., 1976). It has been shown that goniometric measurement can be used as an inexpensive and radiation-free alternative to measuring the $\mathrm{Q}$ angle as accurately as radiography (Chevidikunnan et al., 2017; Stensdotter et al., 2009). However, due to the lack of sufficient reliability coefficients of different methods and measurements, there are no universally accepted normal or abnormal 
values of the $\mathrm{Q}$ angle. This may be due to variations in the measurement procedure (Smith et al., 2008). The $Q$ angle and reference points are first described in 1964 (Brattström, 1964) (Figure 1). He defines the $Q$ angle as the complementary angle formed by the resultant line of force of the quadriceps and a line from the midpoint of the patella (MP) to the center of the tibial tubercle (TT). Although anatomical reference points are clearly defined, the procedure for measuring the $Q$ angle is not standardized, as indicated in the literature. For example, whether the $\mathrm{Q}$ angle "should be measured in quadriceps relax or isometric contraction; should the feet be positioned shoulder-width apart or with the medial malleolus touching; and should the position be standing or supine? ", these questions are not answered (Melicharek et al., 2011). It is reported that any method that increases the reliability and applicability of the $\mathrm{Q}$ angle measurement can be useful for investigating the etiology of PFPS and evaluating the treatment result (Herrington \& Nester, 2004; Smith et al., 2008). The literature indicates there is a need for high-quality studies on female individuals with PFPS.

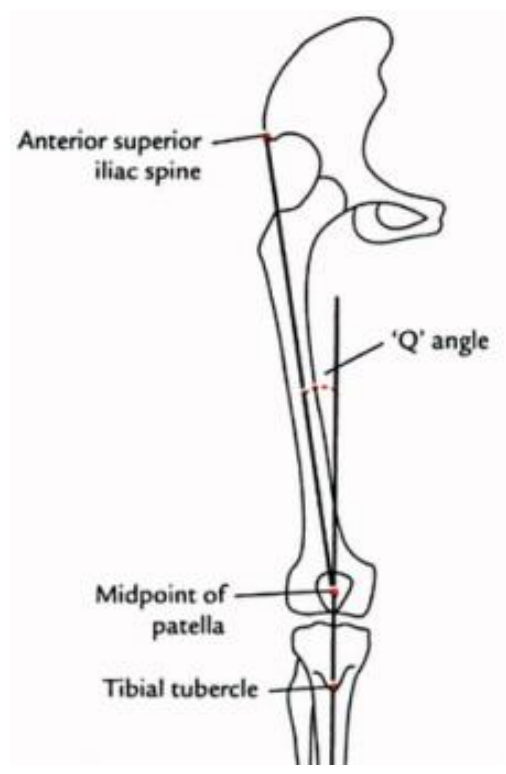

Figure 1. Reference points of $Q$ angle measurement: Anterior superior iliac spine (ASIS), the midpoint of the patella (MP), tibial tubercle (TT).
In our study, the aim is to examine the change of the $\mathrm{Q}$ angle in the relaxed or MVC of the quadriceps in the supine and leg neutralized positions using axial rotation neutralizing apparatus (ARNA) and a manual extendable fixed-arm goniometer in women with PFPS. In this way, our study is trying to contribute to the standardization of $\mathrm{Q}$ angle measurement methods. Besides, the correlation of the $Q$ angle with some of the body parameters is examined. Our results are discussed and presented in the light of the literature.

\section{Methods}

Thirty-four sedentary females with PFPS between the ages of 18 and 50 were included in the study. When determining the age range the lower limit (Tria \& Alicea, 1995), the age of closure of the epiphyseal plates in the knee joint, and for the upper limit the possible degenerative changes in the knee and hip were taken into account (Almeida et al., 2016). Inclusion criteria were unilateral, prepatellar and/or retropaellar knee pain lasting more than 6 weeks, no history of knee trauma, no surgery, no patellar subluxation or dislocation, and no signs of meniscus or ligament lesions. Females were selected due to their higher incidence of PFPS in this population compared to males and due to kinematic differences between the two sexes.

The patients were informed in detail about the study followed by a consent form. Both the consent and the experimental procedure were in accordance with the Declaration of Helsinki and approved by the Ankara Yildırım Beyazıt University Yenimahalle Education and Research Hospital Clinical Research Ethics Committee. Leg length measurement is done with a tape measure in the supine position by measuring the distance between the anterior superior iliac spine (ASIS) and the medial malleolus (Messier et al., 1991). Body mass index (BMI) is calculated from height and weight by the formula: $\mathrm{BMI}=$ 
weight $(\mathrm{kg}) /$ height $^{2}\left(\mathrm{~m}^{2}\right)$. A manual extendable fixed-arm goniometer was used to measure the $\mathrm{Q}$ angle. In addition, the ARNA we developed was used to prevent axial rotation in the leg and for placing all patients in a standard position (Figure 2). The participants were measured under circumstances where the quadriceps was relaxed or maximally contracted. Individuals adopted a supine position with the knees extended and the quadriceps relaxed. The ASIS and the TT were palpated and marked. For the first measurement, the MP was marked on adhesive tape while the quadriceps was relaxed. The measurement was made so that the goniometer's fixed arm showed the ASIS and the moving arm showed the TT (Biedert \& Warnke, 2001; Brattström, 1964; Merchant et al., 2020; Messier et al., 1991). After the adhesive tape was removed, the patients were asked to perform a maximum isometric contraction of the quadriceps. In this state, the MP was marked again on the renewed tape. After a short rest period, the patients were asked to repeat the maximum isometric contraction of the quadriceps and the $\mathrm{Q}$ angle measurement was repeated in the same fashion as the initial measurement.

Statistical analysis of the data was carried out with the help of IBM SPSS version 25.0 package program. Shapiro Wilk, Skewness and Kurtosis, the ratio of standard deviation (sd) / mean, histogram, and Q-Q plot data were used for normality tests. Paired samples student t-test was used for the relation of normally distributed data between dependent groups. Correlation analysis of the data was performed by using Pearson's correlation test for normally distributed and Spearman correlation test for non-normally distributed. The significance level was accepted as $p<.05$ in all analyses.

\section{Results}

Descriptive data of the participants were analysed. The age, height, weight, BMI, and leg length analysis of the patients are shown in Table 1.

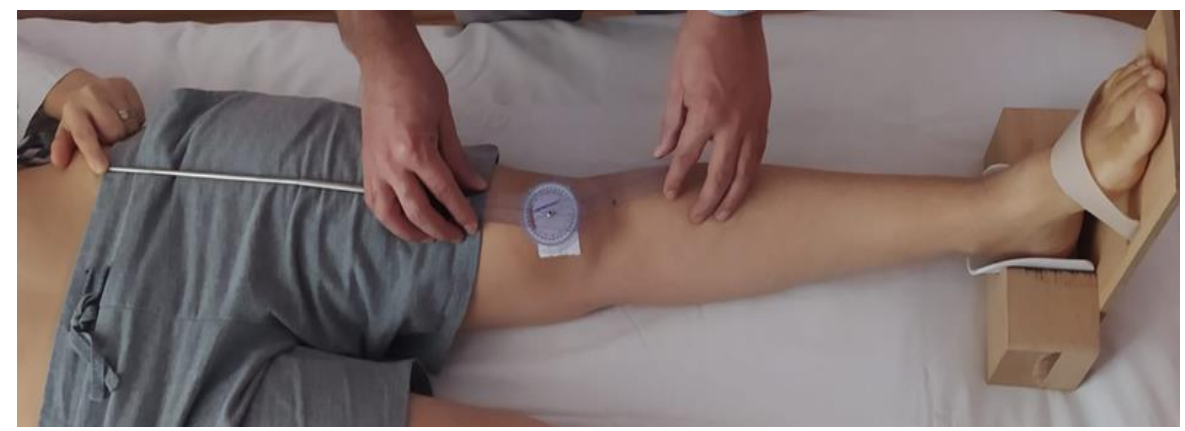

Figure 2. $\mathrm{Q}$ angle measurement technique using ARNA and extendable fixed-arm goniometer.

\begin{tabular}{lc} 
Table 1 & \\
Descriptive data of the participants. & \\
\hline Parameters $(\mathrm{n}=34)$ & Value \\
\hline Age (year) & $27(20-47)^{\mathrm{m}}$ \\
Weight $(\mathrm{kg})$ & $58 \pm 7.97^{\mathrm{n}}$ \\
Height $(\mathrm{cm})$ & $160.88 \pm 5.51^{\mathrm{n}}$ \\
Leg length (cm) & $81.74 \pm 4.29^{\mathrm{n}}$ \\
BMI* $\left(\mathrm{kg} / \mathrm{m}^{2}\right)$ & $21.98(17.55-29.58)^{\mathrm{m}}$ \\
\hline${ }^{*}$ Body Mass Index; ${ }^{\mathrm{m}}$ median $\left(\right.$ min-max); ${ }^{n}$ mean $\pm s d$ &
\end{tabular}


$\mathrm{Q}$ angles of the quadriceps in relaxed and MVC conditions were compared. It was found that the mean of the $\mathrm{Q}$ angle in the quadriceps relaxed position in participants was higher than the mean of the $\mathrm{Q}$ angle in the contracted position $\left(18.44^{\circ} \pm 2.80^{\circ} ; 16.41^{\circ} \pm 2.83^{\circ}\right.$, respectively). The $Q$ angle decreased significantly with contraction $(\mathrm{p}<.01)$ (Table 2$)$.

\section{Table 2}

$\mathrm{Q}$ angles of the participants in two different states of the quadriceps.

\begin{tabular}{lccc}
\hline Parameter $(\mathrm{n}: 34)$ & Relaxed & Contracted & $\mathrm{p}$ \\
\hline Q Angle & $18.44 \pm 2.80$ & $16.41 \pm 2.83$ & $.00^{\gamma}$ \\
\hline${ }^{v}$ Paired samples student $-\mathrm{t}$ & &
\end{tabular}

When the correlation between the $\mathrm{Q}$ angles of the quadriceps and the descriptive characteristics of the participants was examined, it was found no correlation between the age, height, weight, BMI, leg length, and Q angles of the individuals (Table 3).

\section{Table 3}

Correlation between the descriptive parameters of the participants and $Q$ angles $(n=34)$.

\begin{tabular}{lcc}
\hline Variables & Relaxed $\mathrm{r}(\mathrm{p})$ & Contracted $\mathrm{r}(\mathrm{p})^{\mathrm{a}}$ \\
\hline Age (year) & $-.014(0.937)^{\mathrm{a}}$ & $.229(0.193)^{\mathrm{a}}$ \\
Weight $(\mathrm{kg})$ & $.006(0.973)^{\mathrm{b}}$ & $-.048(0.787)^{\mathrm{b}}$ \\
Height $(\mathrm{cm})$ & $.156(0.378)^{\mathrm{b}}$ & $-.008(0.962)^{\mathrm{b}}$ \\
Leg length $(\mathrm{cm})$ & $.289(0.098)^{\mathrm{b}}$ & $.024(0.892)^{\mathrm{b}}$ \\
BMI $\left(\mathrm{kg} / \mathrm{m}^{2}\right)$ & $-.092(0.606)^{\mathrm{a}}$ & $-.072(0.687)^{\mathrm{a}}$ \\
\hline${ }^{a}$ Spearman correlation test; ${ }^{\mathrm{b}}$ Pearson correlation test
\end{tabular}

\section{Discussion}

To be clear, this study does not present a new test but describes a new standard protocol for an old test to make it a useful physical examination technique for assessing the $\mathrm{Q}$ angle.

For patients in the supine and extended knee position, the results of the $\mathrm{Q}$ angle measurements using an extendable fixed arm goniometer and ARNA (in relaxed quadriceps: $18.44^{\circ} \pm 2.80^{\circ}$; in MVC of quadriceps: $16.41^{\circ} \pm$ $2.83^{\circ}$ ) were found to be consistent with the literature. When compared to the literature, the $\mathrm{Q}$ angle measurements of women with relaxed quadriceps in the supine position were similar to the measurements of symptomatic female individuals in the same position (Almeida et al., 2016; Draper et al., 2011; Guerra et al., 1994; Merchant et al., 2020; Shiva Prakash et al., 2019; Woodland \& Francis, 1992) but higher than the mean measurements of asymptomatic female individuals (Aglietti et al., 1983; Choudhary et al., 2019; Draper et al., 2011; Merchant et al., 2020; Raveendranath et al., 2020; Shiva Prakash et al., 2019; Woodland \& Francis, 1992). In Almeida et al.'s evaluation of 22 individuals in the supine position with PFPS, they found a mean $\mathrm{Q}$ angle of $17.8^{\circ} \pm 2.7^{\circ}$ (Almeida et al., 2016). On the other hand, in analyses of 200 individuals (half of them females) in the supine position with their quadriceps relaxed, the mean $\mathrm{Q}$ angle of the female participants was determined to be $14.48^{\circ} \pm 2.02^{\circ}$ (Shiva Prakash et al., 2019). In another study of 257 asymptomatic female individuals, the mean $\mathrm{Q}$ angle was reported to be $15.8^{\circ}$ (Woodland \& Francis, 1992). In a study comparing symptomatic and asymptomatic individuals in a relaxed supine position, the means of $Q$ angle were found to be $20^{\circ}$ and $17^{\circ}$, respectively (Aglietti et al., 1983). Moreover, a study using an extendable fixed-arm goniometer to standardize the $\mathrm{Q}$ angle found a mean angle of $15.9^{\circ} \pm 4.8^{\circ}$ in its measurements of asymptomatic female subjects (Merchant et al., 2020). In a study that measured participants in a relaxed supine position with computed tomography, the patella was more significantly situated in the lateral tilt position of the PFPS group than the healthy group. The authors also concluded that these findings may indicate patellar incompatibility caused by passive and active patellar stabilizers (Esfandiarpour et al., 2018). 
When the quadriceps is contracted when the knee is in extension, that is, there is no patellofemoral contact; the tensile force will want to pull the SIAS and TT in the same direction, so the patella slides laterally. This is proportional to the magnitude of the angle $Q$ (Kuru et al., 2012). If the $Q$ angle exceeds the normal, the lateral pulling force increases. An increased $Q$ angle has been suggested to be an indicator of pathological lateral forces on the patella (Burke et al., 2018; Chhabra et al., 2016; Insall et al., 1976). The greater the $Q$ angle, the greater the lateralization force on the patella, increasing the retropatellar pressure between the lateral facet of the patella and the lateral femoral condyle (Brechter \& Powers, 2002). A $10 \%$ increase in the $Q$ angle results in a $45 \%$ stress increase in the patellofemoral joint (Huberti \& Hayes, 1984). These continuous compressive forces between the femoral condyle and the lateral patellar facet can cause long term degeneration of the patellar joint cartilage resulting in the formation of PFPS (Brechter \& Powers, 2002; Lee et al., 2003).

In our study, the mean $Q$ angle values obtained from MVC of the quadriceps were found to be significantly lower than the values obtained from the relaxed quadriceps position. In a study that investigated how $Q$ angle measurements are affected by the position and contraction of the quadriceps, measurements were made in both supine and standing positions with the quadriceps in relaxed or contracted states. In turn, the measurements of the contracted quadriceps in both positions were found to be significantly lower than those taken of the relaxed quadriceps (Guerra et al., 1994). Belchior et al. analyzed the $Q$ angle measurements of quadriceps that were relaxed and MVC. These measurements were performed with X-rays on women in the supine position. Although the measurements of symptomatic individuals in relaxed and contracted positions were reported to be $21.45^{\circ}$ and $15.8^{\circ}$, respectively, the data from asymptomatic patients were reported to be $17.15^{\circ}$ and $14.5^{\circ}$, respectively (Belchior et al., 2006). In a study performed with magnetic resonance imaging, lateral maltraction was significantly higher in symptomatic patients than in asymptomatic patients (Burke et al., 2018). The greater $Q$ angle and lateralization in symptomatic individuals supports the idea that the $\mathrm{Q}$ angle is inversely proportional to the quadriceps force (Bayraktar et al., 2004; Binder et al., 2001; Byl et al., 2000).

While the relationship between the participants' $\mathrm{Q}$ angle measurements and some of the body parameters (age, weight, BMI, height, leg length) were examined, no correlations between these factors were found. Similarly, studies have indicated that there is no relationship between age and $\mathrm{Q}$ angle in adults (Choudhary et al., 2019; Grelsamer et al., 2005; Khasawneh et al., 2019; Tarawneh et al., 2016). Choudhary et al.'s evaluation of 150 participants reported no relationship between these individuals' $Q$ angles and their ages (Choudhary et al., 2019). Likewise, Tarawneh et al.'s study of 419 people, identified no relationship between body weight and $Q$ angles but did find a positive correlation between individuals' BMIs and $Q$ angles (Tarawneh et al., 2016). Many studies have reported a negative correlation between height and Q angle (Choudhary et al., 2019; Grelsamer et al., 2005; Khasawneh et al., 2019; Kwon, 1999; Tarawneh et al., 2016). Contrary to the literature, the present study identified no correlation between height, leg length, and $Q$ angle. This discrepancy may be due to the similar heights of the current study's the participants.

\section{Conclusions}

According to the results of the data obtained from standardized $Q$ angle measurements using an extendable fixed-arm goniometer and ARNA, the $Q$ angle values decrease in the contracted quadriceps compared to in the 
relaxed quadriceps of patients with PFPS. When measuring, ASIS and TT reference points are fixed. Therefore, the main reason for this reduction is thought to be the lateral shift of the patella with contraction. When clinical $Q$ angle measurement is standardized for both preliminary assessments and evaluations of the effectiveness of treatment, the elimination of leg rotation and the extent of the functional effect of the quadriceps muscle on the $Q$ angle should be considered. The present study was limited in that it contained no control group that included healthy individuals. Further studies are recommended to include more participants, different control groups, and other non-standardized methods.

\section{Acknowledgments}

This research did not receive any specific grant from funding agencies in the public, commercial, or not-for-profit sectors.

\section{Conflicts of Interest}

The authors have no conflicts of interest relevant to this article.

\section{References}

Aglietti, P., Insall, J.N., \& Cerulli, G. (1983). Patellar pain and incongruence. I: Measurements of incongruence. Clin Orthop Relat Res, (176), 217224.

Almeida, G.P.L., Carvalho, A.P.d.M.C., França, F.J.R., Magalhães, M.O., Burke, T.N., \& Marques, A.P. (2016). Q-angle in patellofemoral pain: relationship with dynamic knee valgus, hip abductor torque, pain and function. Rev. Bras. Ortop. (Sao Paulo) (English Edition), 51(2), 181-186.

Bayraktar, B., Yucesir, I., Ozturk, A., Cakmak, A.K., Taskara, N., Kale, A., Demiryurek, D., Bayramoglu, A., \& Camlica, H. (2004). Change of quadriceps angle values with age and activity. Saudi Med J, 25(6), 756-760.

Belchior, A., Arakaki, J., Bevilaqua-Grossi, D., Reis, F., \& Carvalho, P. (2006). Effects in the $Q$ angle measurement with maximal voluntary isometric contraction of the quadriceps muscle. Rev Bras Med Esporte, 12(1), 5e-8e.

Biedert, R., \& Warnke, K. (2001). Correlation between the $\mathrm{Q}$ angle and the patella position: a clinical and axial computed tomography evaluation. Arch Orthop Trauma Surg, 121(6), 346349.

Binder, D., Brown-Cross, D., Shamus, E., \& Davies, G. (2001). Peak torque, total work and power values when comparing individuals with Qangle differences. Isokinet Exerc Sci, 9(1), 27-30.

Boling, M., Padua, D., Marshall, S., Guskiewicz, K., Pyne, S., \& Beutler, A. (2010). Gender differences in the incidence and prevalence of patellofemoral pain syndrome. Scand J Med Sci Sports, 20(5), 725730.

Brattström, H. (1964). Shape of the intercondylar groove normally and in recurrent dislocation of patella: a clinical and $\mathrm{x}$-ray anatomical investigation. Acta Orthop Scand, 35(sup68), 1148.

Brechter, J.H., \& Powers, C.M. (2002). Patellofemoral stress during walking in persons with and without patellofemoral pain. Med Sci Sports Exerc, 34(10), 1582-1593.

Burke, C.J., Kaplan, D., Block, T., Chang, G., Jazrawi, L., Campbell, K., \& Alaia, M. (2018). Clinical utility of continuous radial magnetic resonance imaging acquisition at $3 \mathrm{~T}$ in real-time patellofemoral kinematic assessment: a feasibility study. Arthroscopy, 34(3), 726-733.

Byl, T., Cole, J.A., \& Livingston, L.A. (2000). What determines the magnitude of the $\mathrm{Q}$ angle? A preliminary study of selected skeletal and muscular measures. J Sport Rehabil, 9(1), 26-34.

Chevidikunnan, M.F., Al Saif, A., Pai, H., \& Mathias, L. (2017). Comparing goniometric and radiographic measurement of $\mathrm{Q}$ angle of the knee. Asian Biomed (Res Rev News), 9(5), 631-636.

Chhabra, P.K., Setiya, M., \& Godwin, R. (2016). Quadriceps angle: an important indicator of biomechanical function of lower extremity and its relation with anterior knee pain. Int J Sci Stud, $4(7), 173-176$.

Choudhary, R., Malik, M., Aslam, A., Khurana, D., \& Chauhan, S. (2019). Effect of various 
parameters on Quadriceps angle in adult Indian population. J Clin Orthop Trauma, 10(1), 149-154.

Crossley, K.M., van Middelkoop, M., Callaghan, M.J., Collins, N.J., Rathleff, M.S., \& Barton, C.J. (2016). 2016 Patellofemoral pain consensus statement from the 4th International Patellofemoral Pain Research Retreat, Manchester. Part 2: recommended physical interventions (exercise, taping, bracing, foot orthoses and combined interventions). $\mathrm{Br}$ J Sports Med, 50(14), 844-852.

Draper, C.E., Chew, K.T., Wang, R., Jennings, F., Gold, G.E., \& Fredericson, M. (2011). Comparison of quadriceps angle measurements using short-arm and long-arm goniometers: correlation with MRI. PM $\mathcal{E} R, 3(2), 111-116$.

Esfandiarpour, F., Lebrun, C.M., Dhillon, S., \& Boulanger, P. (2018). In-vivo patellar tracking in individuals with patellofemoral pain and healthy individuals. J Orthop Res, 36(8), 2193-2201.

Grelsamer, R., Dubey, A., \& Weinstein, C. (2005). Men and women have similar $Q$ angles: a clinical and trigonometric evaluation. J. Bone Joint Surg. Am British, 87(11), 1498-1501.

Guerra, J.P., Arnold, M.J., \& Gajdosik, R.L. (1994). Q angle: effects of isometric quadriceps contraction and body position. J Orthop Sports Phys Ther, 19(4), 200-204.

Herrington, L., \& Nester, C. (2004). Q-angle undervalued? The relationship between $\mathrm{Q}$-angle and medio-lateral position of the patella. Clin Biomech (Bristol, Avon), 19(10), 1070-1073.

Huberti, H., \& Hayes, W. (1984). Patellofemoral contact pressures. The influence of q-angle and tendofemoral contact. J Bone Joint Surg Am, 66(5), 715-724.

Insall, J., Falvo, K., \& Wise, D. (1976). Chondromalacia Patellae. A prospective study. J Bone Joint Surg Am, 58(1), 1-8.

Khasawneh, R.R., Allouh, M.Z., \& Abu-El-Rub, E. (2019). Measurement of the quadriceps (Q) angle with respect to various body parameters in young Arab population. PloS one, 14(6).

Kwon, H.-C. (1999). Factors related to Q angle in healthy adults Phys Ther Korea, 6(1), 1-14.
Kuru, İ., Haberal, B., \& Avc1, Ç. (2012). Patellofemoral biomechanics. Journal of the Turkish Society of Orthopaedics and Traumatology, 11(4), 274-280.

Lee, T.Q., Morris, G., \& Csintalan, R.P. (2003). The influence of tibial and femoral rotation on patellofemoral contact area and pressure. J Orthop Sports Phys Ther, 33(11), 686-693.

Melicharek, A., Miller, M.K., \& Hazzard, J. (2011). A comparison of the bilateral, dynamic Q-angle in females. Paper presented at the ISBS-Conference Proceedings Archive.

Merchant, A.C., Fraiser, R., Dragoo, J., \& Fredericson, M. (2020). A reliable Q angle measurement using a standardized protocol. The Knee, 27(3), 934-939.

Messier, S.P., Davis, S.E., Curl, W.W., Lowery, R.B., \& Pack, R.J. (1991). Etiologic factors associated with patellofemoral pain in runners. Med Sci Sports Exerc, 23(9), 1008-1015.

Raveendranath, V., Nachiket, S., Sujatha, N., Priya, R., \& Rema, D. (2020). The quadriceps angle (Q angle) in Indian men and women. Eur J Anat, 13(3), 105-109.

Santos, T.R., Oliveira, B.A., Ocarino, J.M., Holt, K.G., \& Fonseca, S.T. (2015). Effectiveness of hip muscle strengthening in patellofemoral pain syndrome patients: a systematic review. Braz J Phys Ther, 19(3), 167-176.

Shiva Prakash, S., Choudhary, M., \& Manjappa, C. (2019). Influence of gender and bilateral variability of the quadriceps angle ( $Q$ angle) among adults. Int J Orthop Sci, 5(2), 688-691.

Smith, T.O., Hunt, N.J., \& Donell, S.T. (2008). The reliability and validity of the Q-angle: a systematic review. Knee Surg. Sports Traumatol. Arthrosc, 16(12), 1068-1079.

Stensdotter, A.-K., Andersson, P.-I., Rydh, A., \& Häger-Ross, C. (2009). Q-angle variations in standing and supine positions and for different measurement methods in women with and without patellofemoral pain. Adv Physiother, 11(2), 88-96.

Tarawneh, I., AL-Ajoulin, O., Alkhawaldah, A., Kalbouneh, H., \& Hadidi, M. (2016). Normal values of quadriceps angle and its correlation 
with anthropometric measures in a group of Jordanians. J Roy Soc Med, 102(3396), 1-6.

Tria A.J., Alicea J.A. (1995). Embryology and Anatomy of the Patella. In: Scuderi G.R. (eds) The Patella. New York, NY: Springer.
Woodland, L.H., \& Francis, R.S. (1992). Parameters and comparisons of the quadriceps angle of college-aged men and women in the supine and standing positions. Am J Sports Med, 20(2), 208211. 